\title{
Comparison of Lumbar Laminectomy Alone, Lumbar Laminectomy and Fusion, Stand-alone Anterior Lumbar Interbody Fusion, and Stand- alone Lateral Lumbar Interbody Fusion for Treatment of Lumbar Spinal Stenosis: A Review of the Literature
}

\author{
Manan Shah ${ }^{1}$, Bradley Kolb ${ }^{2}$, Emre Yilmaz ${ }^{3}$, Dia R. Halalmeh ${ }^{4}$, Marc D. Moisi ${ }^{1}$ \\ 1. Neurosurgery, Wayne State University, Detroit Medical Center, Detroit, USA 2. Neurosurgery, Rush University \\ Medical Center, Chicago, USA 3. Surgery, Swedish Neuroscience Institute, Seattle, USA 4. Neurosurgery, Detroit \\ Medical Center, Detroit, USA
}

Corresponding author: Dia R. Halalmeh, deaa_h1@yahoo.com

\begin{abstract}
Lumbar spinal stenosis is defined as narrowing of the lumbar spinal canal, which causes compression of the spinal cord and nerves. Spinal stenosis can cause leg pain and potentially back pain that can affect the quality of life. Ultimately, surgical decompression is required to alleviate the symptoms. In this review, we first utilize several important studies to compare lumbar laminectomy alone versus lumbar laminectomy and fusion. We also compare the effectiveness of more novel surgical approaches, stand-alone anterior lumbar interbody fusion (ALIF), and stand-alone lateral lumbar interbody fusion (LLIF). These techniques have their own advantages and disadvantages in which many factors must be taken into account before choosing a surgical approach. In addition, the patient's anatomy and pathology, lifestyle, and desires should be analyzed to help determine the ideal surgical strategy
\end{abstract}

Received 07/29/2019 Review began 07/30/2019 Review ended 09/03/2019 Published 09/18/2019

๑) Copyright 2019 Shah et al. This is an open access article distributed under the terms of the Creative Commons Attribution License CC-BY 3.0., which permits unrestricted use, distribution, and reproduction in any medium, provided the original author and source are credited.
Categories: Neurosurgery

Keywords: spine, lumbar stenosis, lumbar spine fusion, lumbar laminectomy, anterior lumbar interbody fusion, lateral lumbar interbody fusion

\section{Introduction And Background}

Lumbar spinal stenosis is a condition caused by narrowing of the lumbar spinal canal from hypertrophy of facets, hypertrophy of the ligamentum flavum, and/or bulging intervertebral discs, which can lead to compression of spinal nerves, cauda equina, and/or blood vessels [1]. It typically causes leg pain and potentially back pain, particularly when walking (known as intermittent neurogenic claudication), and it can severely affect one's quality of life [2]. It is one of the most common spinal conditions in the elderly (especially older than 60) in the United States, and by 2025, about 64 million elderly will be affected by it $[1,3]$. Along with lumbar stenosis, concomitant lumbar degenerative spondylolisthesis can also occur, which is the slippage of one lumbar vertebra on another. Initial treatment in patients with lumbar stenosis with or without spondylolisthesis is usually conservative management including anti-inflammatory drugs, analgesics, physiotherapy, and epidural infiltration [1]. However, surgery is indicated in patients who develop refractory back and/or leg pain, or even weakness, and those who failed conservative management $[1,4]$. The two main approaches to surgical intervention include decompression of neural structures alone versus decompression plus fusion of adjacent vertebrae. Neural structure decompression via laminectomy has been supplemented more frequently with lumbar fusion to prevent future instability and deformity. In recent years, approximately half the patients who have had surgical intervention for lumbar stenosis have also undergone lumbar fusion [2]. There have been several studies in the past comparing the outcomes of decompressive lumbar laminectomy alone versus laminectomy with added fusion, but the results are conflicting [1]. Since the use of lumbar spine surgery has increased in the past decade, there have also been many new advances and approaches in performing lumbar fusion [2]. The five main approaches to lumbar interbody fusion include posterior lumbar interbody fusion (PLIF), transforaminal lumbar interbody fusion (TLIF), oblique lumbar interbody fusion/anterior to psoas (OLIF/ATP), anterior lumbar interbody fusion (ALIF), and lateral lumbar interbody fusion (LLIF). Each technique has its own advantages and disadvantages but there is no definitive evidence for one approach being better than the other in terms of fusion or clinical outcome [5]. In this article, we review the literature to compare the efficacy of the surgical options for lumbar stenosis, including decompressive lumbar laminectomy alone, lumbar laminectomy and fusion, stand-alone ALIF, and stand-alone LLIF.

\section{Review}

\section{Materials and methods}


Peer reviewed, English-only articles were searched through the PubMed database using search terms 'lumbar stenosis, lumbar laminectomy versus fusion, stand-alone anterior lumbar interbody fusion, stand-alone lateral lumbar interbody fusion'. The inclusion criteria were as follows: original articles regarding the different surgical approaches used to address lumbar stenosis including lumbar laminectomy alone, lumbar laminectomy and fusion, stand-alone ALIF, and stand-alone LLIF. The exclusion criteria included articles that reported none of the previously mentioned correlations, those focusing on other surgical approaches used to treat lumbar stenosis, duplication among the database, and non-English language literature. The articles were then filtered to include full text review articles or clinical trial articles only.

\section{Results}

Over 5,000 articles were retrieved using these initial search terms mentioned previously. After overviewing the abstracts and titles of selected articles and filtering, duplicates were excluded from the selected pool and additional articles were removed after a re-screening of titles. The remaining articles were scrutinized for the required information. After further review, 23 peer-reviewed articles were used for this literature review to discuss lumbar laminectomy alone versus lumbar laminectomy and fusion, stand-alone ALIF, and standalone LLIF as follows:

Decompressive Lumbar Laminectomy Alone vs. Lumbar Laminectomy and Fusion

Many studies have investigated whether decompression with fusion resulted in better outcomes than decompression alone for lumbar spinal stenosis. Forsth et al. conducted the Swedish Spinal Stenosis Study, in which patients were randomized to decompression alone or decompression plus fusion to treat lumbar spinal stenosis with or without degenerative spondylolisthesis [2]. The data showed that there was no significant difference between the two groups with regard to the primary outcome, using the Oswestry Disability Index (ODI) at two years and five years (ODI score ranges from 0 to 100, with higher scores indicating more severe disability) [2]. Even analyzing the subgroup of patients with and without spondylolisthesis did not show any significant difference in outcome between the groups. Visual-analogue scales (VAS) for back and leg pain were secondary outcome measures, and they also did not show any significant difference between the two groups at two years [2]. There were also no significant between-group differences with respect to required follow-up surgery within a mean period of 6.5 years, with $22 \%$ of patients in the fusion group and $21 \%$ of patients in the decompression-alone group [2]. While there were not major between-group differences in terms of efficacy or follow-up surgery, there were important differences in terms of complications and cost of care. In terms of complications, dural tears occurred at the same rate in both groups, but wound infection requiring antibiotics but not debridement was more than twice as common in the fusion group (10\%) compared to the decompression-alone group (4\%). The mean length of hospitalization was almost twice as long in the fusion group (7.4 days versus 4.1 days), and the fusion group also had a longer operative time, greater blood loss, and a greater cost of surgery [2]. Taken together, the results of the Swedish Spinal Stenosis Study favor simple decompression over decompression plus fusion for lumbar stenosis with or without spondylolisthesis.

Ghogawala et al. conducted the Spinal Laminectomy versus Instrumented Pedicle screw fusion (SLIP) study, a randomized controlled study that focused on a homogenous population of patients with nonmobile singlelevel grade 1 spondylolisthesis [6]. The patients were randomized to decompression alone or decompression plus fusion and followed for four years. The primary outcome measure looked at health-related quality of life, which was measured using the Short Form-36 (SF-36) physical component summary score (range, 0 to 100, with higher scores indicating better quality of life) [6]. It was found that at two years after surgery, patients in the fusion group had a significantly greater increase in the SF-36 physical-component summary score than did those in the decompression-alone group. This effect was sustained for three and four years after surgery [6]. However, the ODI score, a secondary measure, did not show a significant difference between the two groups at 2, 3, or 4 years post-surgery. Another important finding was that the reoperation rate over the four-year post-surgical period was $14 \%$ in the fusion group and $34 \%$ in the decompressionalone group; this difference was on the threshold for statistical significance $(P=0.05)[6]$. The reoperations for the decompression-alone group were all for clinical instability and all the reoperations in the fusion group were for the adjacent-level disease [6]. Blood loss, length of procedure, and length of hospital stay were all significantly greater in the fusion group. The authors concluded that lumbar laminectomy plus fusion was associated with a slightly greater but clinically meaningful improvement in physical healthrelated quality of life compared to laminectomy alone [6].

The Swedish Spinal Stenosis study and the SLIP study have conflicting results on the efficacy of decompression alone vs. decompression and fusion, with the latter presenting evidence for the superiority of decompression plus fusion, at least for patients with symptomatic stenosis and nonmobile single-level grade 1 spondylolisthesis. The SLIP study has been criticized for its small sample size, as only 66 patients were randomized [7]. The study also showed no difference in the ODI score between the groups, which questions the conclusion that the fusion group had a better quality of life. The ODI score was also a secondary outcome measure instead of a primary one, which has also been criticized [7]. The $34 \%$ reoperation rate in the decompression-alone group is also surprisingly high, and the surgical technique has therefore been called into question by some authors [7]. The reoperation rate of the fusion group was cited at $14 \%$, which was lower than expected. In Epstein's 2015 and 2016 reviews of old and new literature, an adjacent level disease 
was seen in up to $30 \%$ of patients who underwent fusion, and reoperation rates approached $80 \%$ at five years after surgery [7]. The authors of the SLIP trial argue that ODI scores may become better over time. The change in ODI score from baseline for the two groups at four years was on the verge of statistical significance, with a $p$-value of $0.05[6,8]$. However, they state that the lack of power is a major concern for focusing on ODI scores when analyzing the two groups [8]. On the other hand, the SLIP study authors argue that the Swedish study population was heterogenous and did not identify which patients had instability or the number of levels treated, raising concern that their findings of "no benefit" could have been influenced by these shortcomings [8]. In summary, the Swedish study provides level II evidence stating that there is no difference between the decompression-alone group and the decompression plus fusion group. The SLIP study, on the other hand, provides level I evidence for the efficacy of fusion to improve outcomes and lower reoperation rates compared to the decompressive laminectomy alone for patients with spinal stenosis and nonmobile single-level grade 1 spondylolisthesis [8].

Ahmed et al. also performed a meta-analysis of randomized controlled trials and retrospective and prospective cohort studies looking at decompression alone versus decompression plus fusion for lumbar stenosis. The results showed that decompression plus fusion was found to be 2.55 times better compared to decompression alone in terms of ODI [1]. The decompression plus fusion group was found to be 2.1 times superior to the decompression alone group in terms of the VAS for back pain and 1.4 times superior for leg pain [1]. Overall, the authors concluded that decompression plus fusion is 3.5 times superior to decompression alone in terms of ODI and VAS for back pain and leg pain [1].

\begin{tabular}{|c|c|c|c|}
\hline & Swedish Spinal Stenosis Study & $\begin{array}{l}\text { Spinal Laminectomy versus } \\
\text { Instrumented Pedicle screw fusion trial } \\
\text { (SLIP) study }\end{array}$ & Ahmed et al. meta-analysis \\
\hline ODI & $\begin{array}{l}\text { No significant difference between } \\
\text { decompression alone and } \\
\text { decompression plus fusion }\end{array}$ & $\begin{array}{l}\text { No significant difference between } \\
\text { decompression alone and } \\
\text { decompression plus fusion }\end{array}$ & $\begin{array}{l}\text { Decompression plus fusion } 2.55 \text { times } \\
\text { better }\end{array}$ \\
\hline $\begin{array}{l}\text { VAS for back } \\
\text { pain and leg } \\
\text { pain }\end{array}$ & $\begin{array}{l}\text { No significant difference between } \\
\text { decompression alone and } \\
\text { decompression plus fusion }\end{array}$ & $\begin{array}{l}\text { No significant difference between } \\
\text { decompression alone and } \\
\text { decompression plus fusion }\end{array}$ & $\begin{array}{l}\text { Decompression plus fusion } 2.1 \text { times } \\
\text { better for back pain and } 1.4 \text { times better } \\
\text { for leg pain }\end{array}$ \\
\hline Reoperation & $\begin{array}{l}\text { No significant difference between } \\
\text { decompression alone and } \\
\text { decompression plus fusion }\end{array}$ & $\begin{array}{l}\text { Greater in decompression alone group. } \\
\text { Threshold for statistical significance }(P= \\
0.05)\end{array}$ & N/A \\
\hline $\begin{array}{l}\text { Operative } \\
\text { Time }\end{array}$ & Significantly longer in the fusion group & Significantly longer in the fusion group & N/A \\
\hline Blood Loss & Significantly greater in the fusion group & Significantly greater in the fusion group & N/A \\
\hline $\begin{array}{l}\text { Cost of } \\
\text { Surgery }\end{array}$ & Significantly greater in the fusion group & Significantly greater in the fusion group & N/A \\
\hline $\begin{array}{l}\text { Length of } \\
\text { Hospital } \\
\text { Time }\end{array}$ & Significantly longer in the fusion group & Significantly longer in the fusion group & N/A \\
\hline
\end{tabular}

TABLE 1: Results of the comparison between lumbar laminectomy alone vs laminectomy and fusion in terms of outcome measures

VAS, visual analogue scale; ODI, Oswestry disability index

Yavin et al. performed a meta-analysis on studies comparing nonoperative management, decompression alone, and decompression plus fusion for the degenerative lumbar disease. They concluded that improvements in pain, disability, and satisfaction were greatest in patients undergoing fusion for spondylolisthesis [9]. The complications and reoperative risk limited the role of fusion in patients without spondylolisthesis [9]. Resnick et al. performed a literature review and published guidelines regarding lumbar fusion in patients without spondylolisthesis. They concluded that in the absence of instability, lumbar fusion has not been shown to improve outcomes in patients with isolated lumbar stenosis, and therefore it is not recommended [10]. In summary, there have been many studies comparing decompression alone to decompression and fusion for lumbar stenosis, but they have had varying conclusions. The patient's anatomy, pathology, lifestyle, and desires all need to be taken into account when choosing the best option. 
When lumbar interbody fusion is indicated in a patient, the decision must be made on which approach to utilize safely and effectively. The anterior approach to the lumbar spine for interbody fusion is one of the predominant techniques for the surgical treatment of discogenic lumbar pain [11]. Historically, ALIF has been linked with high intraoperative complications and failure of fusion due to inadequate cages [11]. With the advent of new operative techniques and cages, stand-alone ALIF surgery has been shown to have satisfactory rates of complication and fusion. The advantages of ALIF over other approaches include direct midline view of the disc space and extensive lateral exposure of the vertebral bodies, which allows efficient disc space clearance and maximization of implant size and surface area [5]. It also allows sparing of the posterior spinal muscles and anterolateral psoas muscles, which reduces postoperative pain and disability [5]. Disadvantages include complications such as vascular and visceral injury, and retrograde ejaculation [5]. The overall risk of vascular injury is between $2.2 \%$ to $6.7 \%$, risk of visceral injury is $5 \%$, and the risk of retrograde ejaculation and sympathetic dysfunction is $3 \%[12,13]$. The ALIF procedure is a good option for the L5-S1 level and a reasonable one for the L4-5 level based on the bifurcation of the great vessels [5]. It is not an option for levels above L4-5.

Rao et al. performed a prospective analysis of patients with low-grade spondylolisthesis who underwent stand-alone ALIF. They found that preoperative spondylolisthesis was reduced to $6.4 \%$ postoperatively, and disc height was increased to $175 \%$ of preoperative values, with both differences reaching statistical significance [14]. The VAS pain score improved from 7.6 to 2.2 and ODI improved from $56.9 \%$ to $17.8 \%$, with both differences reaching statistical significance [14]. The radiological fusion rate was $91 \%$. The overall clinical success rate was $93 \%$ [14]. Lammli et al. reviewed a series of patients with degenerative lumbar disc disease who underwent a level 1 or 2 stand-alone ALIF. At two-year follow-up, the ODI and VAS scores significantly improved over presurgical levels in the ALIF patients [15]. No patient experienced intraoperative or major complications. Out of the 118 patients, three had reoperations not related to the adjacent level or fusion site, three had reoperations related to adjacent level disease, and three had pseudoarthrosis at the fusion level [15]. Amaral et al. performed a retrospective single-center study looking at patients with lumbar stenosis and grade 1 spondylolisthesis who underwent L5-S1 stand-alone ALIF. VAS for back pain decreased from 7.4 preoperatively to 4.2 at three months, and VAS for lower limbs decreased from 5.1 preoperatively to 2.8 at three months, with both differences reaching statistical significance [11]. ODI decreased from 44 preoperatively to 31 at three months, which was also statistically significant [11]. Out of 87 patients, two had venous damage intraoperatively, and two had an accidental opening of the peritoneum. There was one patient with postoperative retroperitoneal hematoma and one with incisional hernia, but no patients had retrograde ejaculation [11]. There have not been many studies regarding standalone ALIF surgery for lumbar stenosis but since the advances in interbody cages, it has shown very promising results.

\section{Stand-alone LLIF}

The lateral approach to the lumbar spine is a novel technique described by Ozgur et al. which consists of accessing the disc space via a lateral retroperitoneal and transpsoas surgical corridor $[5,16]$. This approach can be utilized for approaching the lateral spine from the T12-L1 level to the L4-L5 level [5]. It is not suitable for the L5-S1 level because of obstruction by the iliac crests [5,13]. Benefits of LLIF include a large discectomy, bilateral annular release, insertion of large grafts, correction of deformity, and indirect decompression of spinal nerves [17]. Compared to the anterior approach, this approach is less invasive and avoids retraction of the great vessels and sympathetic chain [13]. The lateral approach, unlike the anterior approach, also typically preserves the ligamentous structures including the anterior longitudinal ligament [14,17-19]. Obesity can facilitate the lateral approach to the target disc by pulling the peritoneal contents anteriorly; therefore, resulting in an easier approach through the retroperitoneal corridor [13]. Conversely, obesity can be a barrier to success with the anterior approach used in ALIF. Relative contraindications to LLIF include abnormal or challenging vascular or plexus anatomy as well as previous retroperitoneal surgery $[5,13]$. With LLIF, there are potential risks to the lumbar plexus, psoas muscle, kidneys and bowel, and sufficient care must be taken during the approach to avoid these key structures $[5,13,17]$.

Ahmadian et al. performed a multicenter chart review study for patients who underwent stand-alone minimally invasive LLIF, and 59 patients were ultimately included with pathologies such as degenerative disc disease, spondylolisthesis and scoliosis [20]. The fusion rate was $93 \%$ at 12 months and only two patients needed a reoperation [20]. The VAS improved from 69.1 to 37.8 and ODI improved from 51.8 to 31.8 , with both differences reaching statistical significance [20]. Of note, $70 \%$ of patients had grade 0 subsidence, while $30 \%$ had grade I and II subsidence [20]. Marchi et al. conducted a prospective observational study of 52 patients who underwent stand-alone LLIF for single-level grade I/II spondylolisthesis. Data showed that the mean VAS back scores decreased from 78 to 31 and mean VAS leg scores decreased from 54 to 31, with both differences reaching statistical significance [18]. The mean ODI scores also significantly improved from $66 \%$ to $30 \%$ [18]. Fusion was seen in $86.6 \%$ of levels treated where incomplete bone ingrowth was observed in the remainder; however, pseudoarthrosis was not seen. Postoperatively, there were 10 patients (19.2\%) who presented with psoas weakness and five patients $(9.6 \%$ ) who had anterior thigh numbness, but both conditions resolved within six weeks [18]. There were a total of seven levels (13.5\% of cases) where revision surgery was necessary. There were five revision cases due to high-grade subsidence causing instability or 
restenosis and two revisions because decompression was not achieved [18]. Agarwal et al. performed a retrospective analysis on 55 patients over the age of 70 undergoing a stand-alone LLIF. The ODI score significantly decreased from 46.2 to 31.1 [21]. Five patients needed to undergo revision surgery for graft subsidence [21]. The study concluded that stand-alone LLIF can be safely and effectively performed in the elderly. Watkins et al. found a non-union rate of $19 \%$ per level and $27 \%$ per patient [17]. Nemani et al. performed a retrospective analysis on 117 patients who underwent stand-alone LLIF and found that at 16month follow-up, $10.3 \%$ required revision surgery for posterior decompression, mostly for restenosis [22]. The authors concluded that stand-alone LLIF represents an acceptable procedure in patients with lumbar spinal stenosis.

Laws et al. compared the biomechanical differences between stand-alone ALIF and stand-alone LLIF. Compared to the intact state, stand-alone LLIF significantly reduced the range of motion in flexion, extension, and lateral bending [13,23]. On the other hand, the authors found that stand-alone ALIF did not stabilize motion segments relative to intact state $[13,23]$. Overall, the stand-alone LLIF procedure can be utilized effectively in certain patient populations.

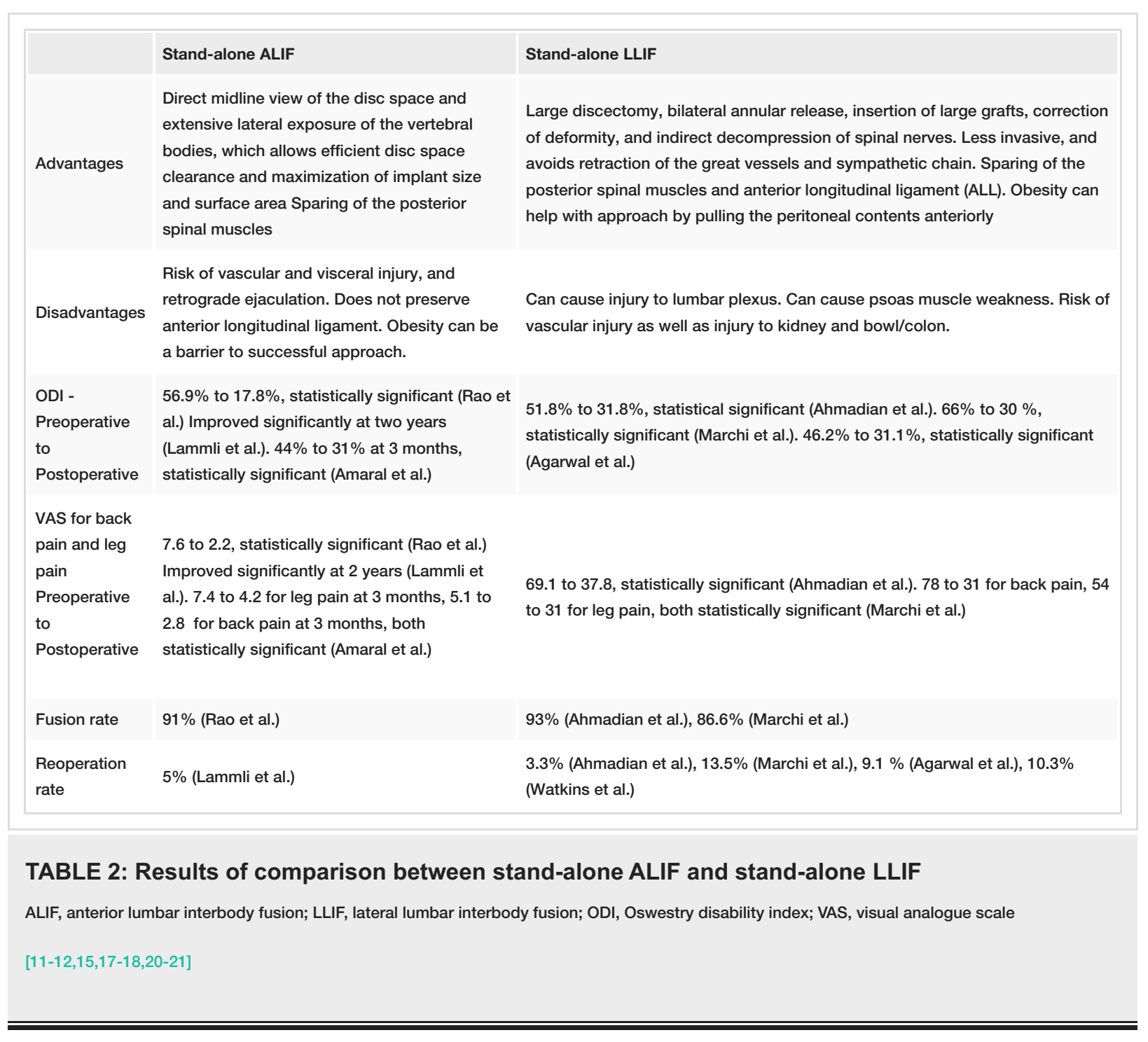

\section{Conclusions}

Lumbar spinal stenosis is a condition that can greatly impact a patient's quality of life. Many studies have been done comparing decompressive lumbar laminectomy alone versus decompression and fusion for lumbar stenosis. In general, lumbar laminectomy alone has been shown to be better for patients with lumbar stenosis in the absence of instability. Studies have shown that lumbar decompression and fusion has been better in patients with spondylolisthesis. In patients who require a fusion, stand-alone ALIF has shown promising results due to the recent advancements in cages, especially at the L4-5 and L5-S1 levels. Standalone LLIF has also shown to be an acceptable procedure for lumbar spinal stenosis. Overall, each technique for the treatment of lumbar stenosis has its own advantages and disadvantages. The individual patient, pathological process, and his or her anatomy must be reviewed to decide which surgical approach is the best. 


\section{Additional Information \\ Disclosures}

Conflicts of interest: In compliance with the ICMJE uniform disclosure form, all authors declare the following: Payment/services info: All authors have declared that no financial support was received from any organization for the submitted work. Financial relationships: All authors have declared that they have no financial relationships at present or within the previous three years with any organizations that might have an interest in the submitted work. Other relationships: All authors have declared that there are no other relationships or activities that could appear to have influenced the submitted work.

\section{References}

1. Ahmed SI, Javed G, Bareeqa SB, et al.: Comparison of decompression alone versus decompression with fusion for stenotic lumbar spine: a systematic review and meta-analysis. Cureus. 2018, 10:e3135. 10.7759/cureus.3135

2. Försth P, Ólafsson G, Carlsson T, et al.: A randomized, controlled trial of fusion surgery for lumbar spinal stenosis. N Engl J Med. 2016, 374:1413-1423. 10.1056/NEJMoa1513721

3. Wu AM, Tong TJ, Wang XY: A rethink of fusion surgery for lumbar spinal stenosis . J Evid Based Med. 2016, 9:166-169. 10.1111/jebm.12215

4. Kelleher MO, Timlin M, Persaud O, Rampersaud YR: Success and failure of minimally invasive decompression for focal lumbar spinal stenosis in patients with and without deformity. Spine. 2010, 35:E981-987. 10.1097/BRS.0b013e3181c46fb4

5. Mobbs RJ, Phan K, Malham G, Seex K, Rao PJ: Lumbar interbody fusion: techniques, indications and comparison of interbody fusion options including PLIF, TLIF, MI-TLIF, OLIF/ATP, LLIF and ALIF. J Spine Surg. 2015, 1:2-18. 10.3978/j.issn.2414-469X.2015.10.05

6. Ghogawala Z, Dziura J, Butler WE, et al.: Laminectomy plus fusion versus laminectomy alone for lumbar spondylolisthesis. N Engl J Med. 2016, 374:1424-1434. 10.1056/NEJMoa1508788

7. Epstein NE: Commentary on: laminectomy plus fusion versus laminectomy alone for lumbar spondylolisthesis by Ghogawala Z, Dziura J, Butler WE, Dai F, Terrin N, Magge SN, et al. NEJM 2016;374 (15):1424-34. Surg Neurol Int. 2016, 7:S644-s647. 10.4103/2152-7806.191061

8. Ghogawala Z, Resnick DK, Glassman SD, Dziura J, Shaffrey CI, Mummaneni PV: Achieving optimal outcome for degenerative lumbar spondylolisthesis: randomized controlled trial results. Neurosurgery. 2017, 64:4044. 10.1093/neuros/nyx207

9. Yavin D, Casha S, Wiebe S, et al.: Lumbar fusion for degenerative disease: a systematic review and metaanalysis. Neurosurgery. 2017, 80:701-715. 10.1093/neuros/nyw162

10. Resnick DK, Watters WC, Mummaneni PV, et al.: Guideline update for the performance of fusion procedures for degenerative disease of the lumbar spine. Part 10: lumbar fusion for stenosis without spondylolisthesis. J Neurosurg Spine. 2014, 21:62-66. 10.3171/2014.4.SPINE14275

11. Amaral R, Ferreira R, Marchi L, Jensen R, Nogueira-Neto J, Pimenta L: Stand-alone anterior lumbar interbody fusion - complications and perioperative results. Rev Bras Ortop. 2017, 52:569-574. 10.1016/j.rboe.2017.08.016

12. Rao PJ, Loganathan A, Yeung V, Mobbs RJ: Outcomes of anterior lumbar interbody fusion surgery based on indication: a prospective study. Neurosurgery. 2015, 76:7-24. 10.1227/NEU.0000000000000561

13. Winder MJ, Gambhir S: Comparison of ALIF vs. XLIF for L4/5 interbody fusion: pros, cons, and literature review. J Spine Surg. 2016, 2:2-8. 10.21037/jss.2015.12.01

14. Rao PJ, Ghent F, Phan K, Lee K, Reddy R, Mobbs RJ: Stand-alone anterior lumbar interbody fusion for treatment of degenerative spondylolisthesis. J Clin Neurosci. 2015, 22:1619-1624. 10.1016/j.jocn.2015.03.034

15. Lammli J, Whitaker MC, Moskowitz A, et al.: Stand-alone anterior lumbar interbody fusion for degenerative disc disease of the lumbar spine: results with a 2-year follow-up. Spine. 2014, 39:E894-901. 10.1097/BRS.0000000000000393

16. Ozgur BM, Aryan HE, Pimenta L, Taylor WR: Extreme Lateral Interbody Fusion (XLIF): a novel surgical technique for anterior lumbar interbody fusion. Spine J. 2006, 6:435-443. 10.1016/j.spinee.2005.08.012

17. Robert Watkins IV, Robert Watkins III, Hanna R: Non-union rate with stand-alone lateral lumbar interbody fusion. Medicine (Baltimore). 2014, 93(29):e275. 10.1097/MD.0000000000000275

18. Marchi L, Abdala N, Oliveira L, Amaral R, Coutinho E, Pimenta L: Stand-alone lateral interbody fusion for the treatment of low-grade degenerative spondylolisthesis. Scientific World Journal. 2012, 456346:2012. $10.1100 / 2012 / 456346$

19. Pimenta L, Oliveira L, Schaffa T, Coutinho E, Marchi L: Lumbar total disc replacement from an extreme lateral approach: clinical experience with a minimum of 2 years' follow-up. J Neurosurg Spine. 2011, 14:3845. 10.3171/2010.9.SPINE09865

20. Ahmadian A, Bach K, Bolinger B, Malham GM, Okonkwo DO, Kanter AS, Uribe JS: Stand-alone minimally invasive lateral lumbar interbody fusion: multicenter clinical outcomes. J Clin Neurosci. 2015, 22:740-746. 10.1016/j.jocn.2014.08.036

21. Agarwal N, Faramand A, Alan N, Tempel ZJ, Hamilton DK, Okonkwo DO, Kanter AS: Lateral lumbar interbody fusion in the elderly: a 10-year experience. J Neurosurg Spine. 2018, 29:525-529. 10.3171/2018.3.SPINE171147

22. Nemani VM, Aichmair A, Taher F, et al.: Rate of revision surgery after stand-alone lateral lumbar interbody fusion for lumbar spinal stenosis. Spine. 2014, 39:E326-331. 10.1097/BRS.0000000000000141

23. Laws CJ, Coughlin DG, Lotz JC, Serhan HA, Hu SS: Direct lateral approach to lumbar fusion is a biomechanically equivalent alternative to the anterior approach: an in vitro study. Spine. 2012, 37:819-825. 10.1097/BRS.0b013e31823551aa 